$57^{\text {ème }}$ Congrès de la SFMBCB, 03002 (2011)

DOI:10.1051/sfmbcb/20115703002

(C) Owned by the authors, published by EDP Sciences, 2011

\title{
Implication de la sphingomyélinase neutre de type 2 dans l'ostéogenèse et la formation des vésicules de minéralisation
}

\author{
Garoby-Salom $\mathrm{S}^{1,2}$, Devillard $\mathrm{R}^{1,2}$, Mucher $\mathrm{E}^{1,2}$, Salvayre $\mathrm{R}^{1,2}$, Nègre-Salvayre $\mathrm{A}^{1,2}$ \\ ${ }^{1}$ INSERM U858, CHU Rangueil, Toulouse, France \\ ${ }^{2}$ Faculté de Médecine, Université Paul Sabatier, Toulouse, France
}

Introduction : Des travaux récents insistent sur le rôle important des lipides dans la minéralisation des os et des dents. La sphingomyéline est un composant majeur des rafts lipidiques et des vésicules formés à partir de la membrane plasmique; ceux-ci ont un rôle essentiel dans la minéralisation de la matrice extracellulaire. Les souris "fro" (fragilitas ossium) homozygotes présentent un déficit de l'activité sphingomyélinase neutre de type 2 (nSMase2) résultant d'une mutation du gène smpd3 (Aubin 2005). Ces souris ont un phénotype de type osteogenesis/dentinogenesis imperfecta avec une très grande fragilité osseuse néonatale et un sévère retard de croissance des os longs. La nSMase2 joue un rôle-clé dans la prolifération, la différenciation et l'apoptose cellulaires induites par les agents de stress (Obeid 2008), mais son rôle dans l'ostéogenèse n'est pas connu. Le phénotype des souris fro suggère que la nSMase joue un rôle majeur dans l'ostéo/dentinogenèse. Le but de ce travail est d'étudier l'implication de la nSMase2 et des sphingolipides dans l'ostéogenèse et la minéralisation des vésicules de matrice extracellulaire.

Matériels et méthodes : Les études sont réalisées sur des préostéoblastes MC3T3-E1 dont la différenciation est induite par l'addition d'acide ascorbique, de $\beta$-glycérophosphate et de dexaméthasone. La différenciation en ostéoblastes est étudiée, après 15 à 20 jours, par la mesure de l'activité phosphatase alcaline; la formation des vésicules de minéralisation par coloration au rouge d'alizarine et caractérisée par immunocytochimie avec des anticorps antiannexine 2. L'activité enzymatique de la nSMase2 est mesurée par hydrolyse de sphingomyéline radioactive. L'induction des facteurs de transcription (Runx2, osterix), de COL1A1 et de l'ostéonectine sont étudiés en Q-PCR (quantitative polymerase chain reaction). Résultats : Les agents de différenciation induisent une activation rapide de nSMase 2 qui est bloquée par un inhibiteur pharmacologique spécifique, le GW4869. L'induction des facteurs de transcription Runx2, osterix et ostéonectine, observée après 4 et 6 jours de différenciation, n'est pas inhibée par le GW4869. A l'inverse, le GW4869 bloque la formation de vésicules de minéralisation colorées à l'alizarine, ainsi que l'activité phosphatase alcaline. Les études en immunocytochimie indiquent que la forte expression d'annexine 2 dans les vésicules de minéralisation est inhibée par le GW4869.

Discussion : Ces résultats montrent que la nSMase2 est activée par les agents de différenciation ostéoblastique. La voie des sphingolipides ne serait pas impliquée dans l'induction des facteurs de transcription de l'ostéogenèse, mais contribuerait à la formation des vésicules de minéralisation. Cette dernière est stoppée par l'inhibiteur de nSMase, le GW4869. Ces résultats obtenus chez la souris fro, dont la nSMase 2 est dysfonctionnelle, constituent une première approche pour la compréhension du rôle de nSMase 2 dans l'ostéogenèse.

Correspondance : sandra.garoby-salom@inserm.fr 